\title{
Evaluating the Approaches of Small Area Estimation Using Poverty Mapping Data
}

\author{
Md. Mizanur Rahman', Deluar J. Moloy ${ }^{2}$ and Md. Sifat Ar Salan ${ }^{3}$
}

\begin{abstract}
Nowadays, estimation demand in statistics is increased worldwide to seek out an estimate, or approximation, which may be a value which will be used for various purpose, albeit the input data could also be incomplete, uncertain, or unstable. The development of different estimation methods is trying to provide most accurate estimate and estimation theory deals with finding estimates with good properties. The demand of small area estimation (SAE) method has been increasing rapidly around the world because of its reliability compared to the traditional direct estimation methods, especially in the case of small sample size. This paper mainly focuses on the comparison of several indirect small area estimation methods (poststratified synthetic, SSD and EB estimates) with traditional direct estimator based on a renowned data set. Direct estimator is approximately unbiased but SSD and Post-stratified synthetic estimator is extreme biased. To cope up the problem, we conduct another model-based estimation procedure namely Empirical Bayes (EB) estimator, which is unbiased and compare them using their coefficient of variation (CV). To check the model assumption, we used Q-Q plot as well as a Histogram to confirm the normality, bivariate correlation, Akaike information criterion (AIC).
\end{abstract}

JEL classification numbers: C13, C51, C51.

Keywords: Small Area Estimation, Direct Estimation, Indirect Estimation, Empirical Bayes Estimator, Poverty Mapping.

${ }^{1}$ Department of Statistics, Mawlana Bhashani Science and Technology University, Bangladesh.

2 Department of Statistics, Mawlana Bhashani Science and Technology University, Bangladesh.

${ }^{3}$ Department of Statistics, Mawlana Bhashani Science and Technology University, Bangladesh.

Article Info: Received: February 1, 2021. Revised: May 5, 2021.

Published online: May 10, 2021. 


\section{Introduction}

Sample survey is a method of data collection with several advantages, such as saving time, money, and energy. Sample survey usually produced reliable estimated value usually direct estimate for mean or total of variable of interest for large areas or domain. Nevertheless, if there is a small area, i.e., if the sample is not large enough for some context, the result can be unacceptably large standard errors if it is based only on direct survey estimators and data from the sample field only. So to develop more reliable estimated value, it is important to use small area statistics. In recent years, interest from both the public and private sectors for accurate estimates for a specific region or area has grown.

The word "small area" generally refers to a population for which accurate statistics of interest cannot be produced, due to certain constraints of the available data. Small area comprises a geographic area such as states, counties, districts, or sub-districts and population community such as age, ethnicity, or gender (S Hariyanto, 2018). Some of the other words that are used as a synonym for a small area include "small domain," "minor domain," "local area," "small sub-domain" (Rao 2003).

Finally, it can be define that small area estimation (SAE) is a statistical technique to estimate the accurate approximation or estimate in the small geographical area where the sample size is very small or even equal to zero.

In situations where direct estimates cannot be disseminated due to unsatisfactory accuracy, an ad hoc collection of methods, called methods for small area estimating (SAE), is necessary to overcome the problem.

These methods are usually referred to as indirect estimators since they handle poor information from sample information belonging to other domains for each domain borrowing strength, resulting in an increase in the effective sample size for each small area.

The growing requirement for more timely and accurate information, along with the high cost of interviews, frequently leads to comprehensive use of survey data. In fact, survey data is used many times to generate estimates in smaller domains or areas than those for which the survey was originally planned. A direct estimator, relying solely on the survey data coming from that area, maybe very inefficient for a region with a low sample size.

This sample size limitation prevents statistical figures from being produced at the demanded level and therefore limits the availability of statistical information to the public or the specific user. By comparison, through increasing the effective sample size, an indirect estimator for a region often uses external data from other areas to improve performance. Among indirect estimators, we consider the ones based on explicit models of regression, called model based estimators. These estimators are focused on the presumption of a constant link between the target variable and certain explanatory variables through areas. The common parameters of the model are estimated using the whole set of sample data, which often leads to small area estimators with significantly better efficiency than direct estimators as long because the assumptions of the model hold. Thus, these strategies include statistical figures 
at a much-disaggregated level without raising the area-specific sample sizes and hence without raising the survey cost.

The key purposes of this research are to evaluate and comparison of various small area estimation approaches using poverty mapping data. In this paper, we use several table and figure to compare these methods.

Research methods define as the technique of strategy those are used for conduction of a research. All methods which are used by the researcher during the course of studying his research problem are termed as research method. In this paper, we will use the following methodologies:

1) Horvitz-Thompson (H-T) estimation use for direct estimation.

2) Post-stratified synthetic indirect estimator use based on statistical approach on implicit models.

3) Empirical Bayes (EB) method indirect estimator use based on statistical approach on explicit models.

4) Mean square error (MSE).

For checking the model assumption, we use the following method:

1. Q-Q plot, as well as Histogram, used to confirm the normality assumption.

2. Bivariate correlation.

3. Akaike information criterion (AIC).

\section{Previous work on poverty mapping indicator}

Poverty maps are an important source of information on the regional distribution of poverty and are currently used to support regional policy making and to allocate funds to local jurisdictions. Good examples are the poverty and inequality maps produced by the World Bank for many countries all over the world. Some previous work on poverty mapping using small area estimation are given below:

One paper (Novi Hidayat Pusponegoro, 2019) seeks to compare the SAE, Spatial SAE and Geo-additive model for calculating a sub-district average per capita income using data from the 2017 Bangka Belitung Province Poverty Survey. The paper's findings are the Geo-additive is the best fit model based on AIC, so the most important part of modeling is the form of relationship between response and covariate.

The research (Mai M. Kamal El Saied, 2019) is to study the SAE procedures for estimating the Egyptian provinces ' mean income and poverty indicators. They demonstrated the direct estimators for mean income and poverty indicators for all provinces. This research also applies the empirical best / Bayes (EB) and pseudoempirical best / Bayes (PEB) approaches focused on unit level -nested error- models for estimating mean income and (FGT) deprivation indices for Egyptian border provinces with (2012-2013) data from the IECS. For comparative purposes, the (MSEs) and coefficient of variance (C. Vs) are determined.

Results (Hukum Chandra, 2018) in district-specific values suggest that the approximate assessments of the proportion of poor households in each district are unreliable, with CVs ranging from $13.33 \%$ to $64 \%$, with an average of $24.69 \%$. The 
CVs of the EBP estimates range from $12.96 \%$ to $37.27 \%$, with $21.19 \%$ on average. It also noted that the direct estimates CVs are greater than 20 percent (30 percent) in 22 (9) of the 38 districts. However, out of the 38 districts, the model-based estimates are greater than 20 per cent (30 per cent) in 20 (3).

The paper (Mai M. Kamal El Saied, 2019) approximate mean income indicates that for all provinces, PEB and EB divided by regional sample sizes have no noticeable differences except for the third of sample size (Red Sea), the PEB therein is greater than the EB. The C.Vs for PEB are smaller than the C.Vs for EB in all selected provinces except the sec for PEB on it is greater than the C.V for EB. The estimated C.Vs are still under $15 \%$ for both methods in all selected provinces. EB estimates for poverty incidence and poverty gap are smaller than PEB for all provinces. Additionally, that the differences are large in three provinces (Matruh, North Sinai and South Sinai), and are small in two of them (Red Sea and New Valley). Estimated poverty rate and poverty gap figures for C.Vs for EB, as predicted.

Paper (Molina, 2009) showed that the estimated CVs of direct estimators of poverty incidences exceeded the level of $10 \%$ for 78 (out of the 104) domains, while those of the EB estimators exceeded this level for only 28 domains. If we increase the level to $20 \%$, then the direct estimators have a greater CV for 17 domains but the CV of EB estimators exceeded $20 \%$ only for the first domain.

In March 2017, the Province of Yogyakarta Special Region (DIY Province) had a poverty line above the national average of IDR 374,009, a proportion of poor people $(13.03 \%)$ and Gini coefficient (0.432) (IDR 374,478; 10.64\%; 0.393). The outcome of the 2017 happiness index indicates that DIY Province's position (72.93\%) is higher than the national happiness index average (70.69\%). For 2017, the dispersal between the index of satisfaction and the proportion of poor people for Indonesia indicates that DIY Province is on the first quadrant. It reflects the high level of happiness as well as the high percentage of the poor. To assess the spatial characteristics of deprivation and happiness profiles in DIY Province, a small area estimate approach developed by Elbers et al. (known as the ELL method) is used. This study utilized data from the village census (Podes) 2018; Susenas March 2017 and SPTK 2017 as data from the survey. There are twenty-three variables for households and another five variables that are significant to urban and rural provincial models of poverty and happiness. Rural regency areas are dominated by a high profile of poverty (FGT0 0.0491-0.1076), low profile of happiness (FTG0 0.0087-0.0124), and inequality of happiness (Gini index 0.0847-0.0923). Low deprivation (FTG0 0.0082-0.0491), high satisfaction level (FTG0 0-0.0087), and total income equality (Gini index 0.3048-0.3604) and happiness levels (Gini index 0.0624-0.0847) dominate the urban regency regions. Yogyakarta City has the happiest and wealthiest profiles, while the urban regency area of Gunung Kidul has perfect income and happiness profile equality (Shafiera Rosa El-Yasha, 2019).

This paper (V.Y., Sundara, 2017) introduced an approach to the impact of the auxiliary variable on the clustering region by believing parallels occur between specific areas. All estimates were determined based on the relative bias and root mean error of the squares. The simulation result showed that the proposed approach 
can enhance model's ability to estimate non-sampled area. The suggested model was applied to estimate poverty measures in regency and city of Bogor, West Java, Indonesia at sub-districts level. The outcome of case study is smaller than the theoretical model relative root means squares error estimation of empirical Bayes with knowledge cluster.

\section{Sources of data}

In this research our aim is to compare some techniques of small area estimation. Such that we use secondary data taken from R package "sae". The name of the collected data set "incomedata" which was a Synthetic data on income and other related variables for Spanish 52 provinces. This is a data frame with 17199 observations with 21 variables. We also use three identifier such as "sizeprov" containing the population size for domains in data set incomedata, "sizeprovedu" population sizes by level of education for domains in data set incomedata and "Xoutsamp" containing the values of $\mathrm{p}$ auxiliary variables for out-of-sample units within domains of data set incomedata.

The data set incomedata contains synthetic unit-level data on income and other sociological variables in the Spanish provinces. These data have been obtained by simulation. Therefore, conclusions regarding the levels of poverty in the Spanish provinces obtained from these data are not realistic. We will use the following variables from the data set: province name (provlab), province code (prov), income (income), sampling weight (weight), education level (educ), labor status (labor), and finally the indicators of each of the categories of educ and labor.

\section{Main Results}

In this study, we used poverty mapping data of Spanish provinces to analyze several simple estimates namely direct estimates, post-stratified synthetic estimates with education levels as post-strata, SSD estimates obtained from the com-position of direct and post-stratified synthetic estimates. Also, we calculate the EB estimator considering the auxiliary variable from the out of sample. The poverty incidence for a province is the province mean of a binary variable taking value 1 when person's income is below a given poverty line and 0 otherwise. Binary variable could use for calculate the direct estimate easily applying usual theory. In this research, we used R, SPSS and Excel as the analysis tools. First, we read the income data set which included the data for each individual and the data sets sizeprov the population sizes and sizeprovedu sizes by education level, respectively.

Considered poverty line $Z=6557.143$ 
Table 1: Frequency distribution of poverty incidence

\begin{tabular}{|c|c|c|}
\hline & Frequency & Percentage (\%) \\
\hline Poor $($ Income $<\mathrm{Z})$ & 3841 & 22.333 \\
\hline Not poor & 13358 & 77.667 \\
\hline Total & 17199 & 100 \\
\hline
\end{tabular}

Maximum number of people about 13358 are not below the poverty line $(\mathrm{Z})$ that is $77.667 \%$ people are not poor. And about 3841 people that is $22.333 \%$ people are below the poverty line i.e., they are poor.

Table 2: Sorted Combination of Direct (DIR), Post-stratified synthetic and Sample size dependent (SSD) according to each province (Sorted by decreasing sample size)

\begin{tabular}{|c|c|c|c|c|}
\hline Province & Sample Size & DIR $\times \mathbf{1 0 0}$ & PSYN.educ $\times \mathbf{1 0 0}$ & SSD $\times \mathbf{1 0 0}$ \\
\hline Barcelona & 1420 & 29.81253 & 21.59556 & 29.81253 \\
\hline Madrid & 944 & 18.21821 & 20.28249 & 18.25089 \\
\hline Murcia & 885 & 17.70317 & 22.50054 & 17.72239 \\
\hline Oviedo & 803 & 26.06401 & 22.00916 & 26.06401 \\
\hline Valencia & 714 & 21.36068 & 21.32963 & 21.36054 \\
\hline Baleares & 634 & 9.999792 & 21.71882 & 10.4024 \\
\hline Navarra & 564 & 16.19077 & 20.92992 & 16.22866 \\
\hline Zaragoza & 564 & 10.03458 & 21.17064 & 10.03458 \\
\hline Alicante & 539 & 20.7851 & 21.26954 & 20.7851 \\
\hline Vizcaya & 524 & 21.69447 & 20.44194 & 21.69447 \\
\hline RiojaLa & 510 & 25.81181 & 22.40296 & 25.78924 \\
\hline CorunaLa & 495 & 25.34755 & 21.76006 & 25.23624 \\
\hline Badajoz & 494 & 21.55389 & 22.35924 & 21.55389 \\
\hline Sevilla & 482 & 20.50304 & 21.74189 & 20.58245 \\
\hline PalmasLas & 472 & 16.65184 & 21.809 & 16.65184 \\
\hline Pontevedra & 448 & 18.54907 & 21.86237 & 18.54907 \\
\hline Santander & 434 & 34.24443 & 21.56598 & 34.07708 \\
\hline Cadiz & 398 & 14.88735 & 22.51448 & 14.88735 \\
\hline Tenerife & 381 & 18.42962 & 21.96155 & 19.17768 \\
\hline Malaga & 379 & 22.91846 & 22.51928 & 22.90551 \\
\hline Valladolid & 299 & 19.29233 & 20.98068 & 19.29233 \\
\hline Guipuzcoa & 285 & 23.69055 & 20.76857 & 23.66709 \\
\hline Caceres & 282 & 27.03132 & 22.23249 & 26.44514 \\
\hline Toledo & 275 & 12.55338 & 23.14442 & 12.57643 \\
\hline CiudadReal & 250 & 20.92153 & 23.23302 & 20.92153 \\
\hline Ceuta & 235 & 19.7248 & 22.81006 & 19.7248 \\
\hline Jaen & 232 & 31.2942 & 22.93972 & 31.2942 \\
\hline Cordoba & 224 & 29.97571 & 22.91798 & 29.51045 \\
\hline Leon & 218 & 18.80157 & 22.93115 & 19.22223 \\
\hline & & & & \\
\hline
\end{tabular}




\begin{tabular}{|c|c|c|c|c|}
\hline Granada & 208 & 31.72734 & 22.39243 & 30.97619 \\
\hline Almeria & 198 & 26.76398 & 23.02936 & 26.76398 \\
\hline Melilla & 180 & 19.10912 & 22.00697 & 19.43014 \\
\hline Albacete & 173 & 14.05924 & 22.67562 & 14.30411 \\
\hline Lugo & 173 & 37.71872 & 23.94922 & 37.58235 \\
\hline Burgos & 168 & 21.41315 & 22.35331 & 21.41315 \\
\hline Salamanca & 164 & 16.10451 & 21.9324 & 16.76284 \\
\hline Gerona & 142 & 18.33742 & 21.596 & 18.85399 \\
\hline Tarragona & 134 & 32.03544 & 22.51761 & 29.51279 \\
\hline Lerida & 130 & 15.55959 & 23.89632 & 15.55959 \\
\hline Orense & 129 & 22.79961 & 23.58691 & 22.96765 \\
\hline Huelva & 122 & 12.58345 & 22.35069 & 13.442 \\
\hline Castellon & 118 & 17.5982 & 21.91192 & 18.73778 \\
\hline Huesca & 115 & 24.10761 & 23.10616 & 23.98812 \\
\hline Zamora & 104 & 30.02744 & 26.17055 & 30.02744 \\
\hline Alava & 96 & 25.50373 & 20.7788 & 24.08931 \\
\hline Cuenca & 92 & 26.33406 & 24.83639 & 26.13496 \\
\hline Guadalajara & 89 & 17.90818 & 22.59389 & 18.78456 \\
\hline Palencia & 72 & 30.16607 & 23.63212 & 29.39216 \\
\hline Teruel & 72 & 27.36424 & 22.89205 & 26.70145 \\
\hline Avila & 58 & 5.5122 & 22.8933 & 10.28835 \\
\hline Segovia & 58 & 22.262 & 22.67927 & 22.33761 \\
\hline Soria & 20 & 2.541207 & 23.10395 & 13.14019 \\
\hline & & & & \\
\hline
\end{tabular}

For simplification, the estimated values of each estimator in the Table 2 is multiplied by 100. This table shows that direct estimates and SSD estimates are very similar. The estimated value of these two methods are fluctuate decreases as sample size decreases and they are more slightly more unstable. It is noticeable that when sample size is large the SSD estimator treated as a direct estimator, but it is increases when sample size was small such as Table 2 shows that in "Soria" province (sample size $=20$ ) direct estimated value was 2.541207 which increases to 13.14019 in SSD estimate, in "Avila" province 5.5122 (direct) tern in to 10.28835 (SSD) and others values are approximately similar in both direct and SSD estimates. Otherwise, the synthetic estimator has a bigger contribution. However, the post-stratified synthetic estimates appear to be too stable, giving practically the same values for all provinces. It can be shown more clearly in the following Figure 1. 


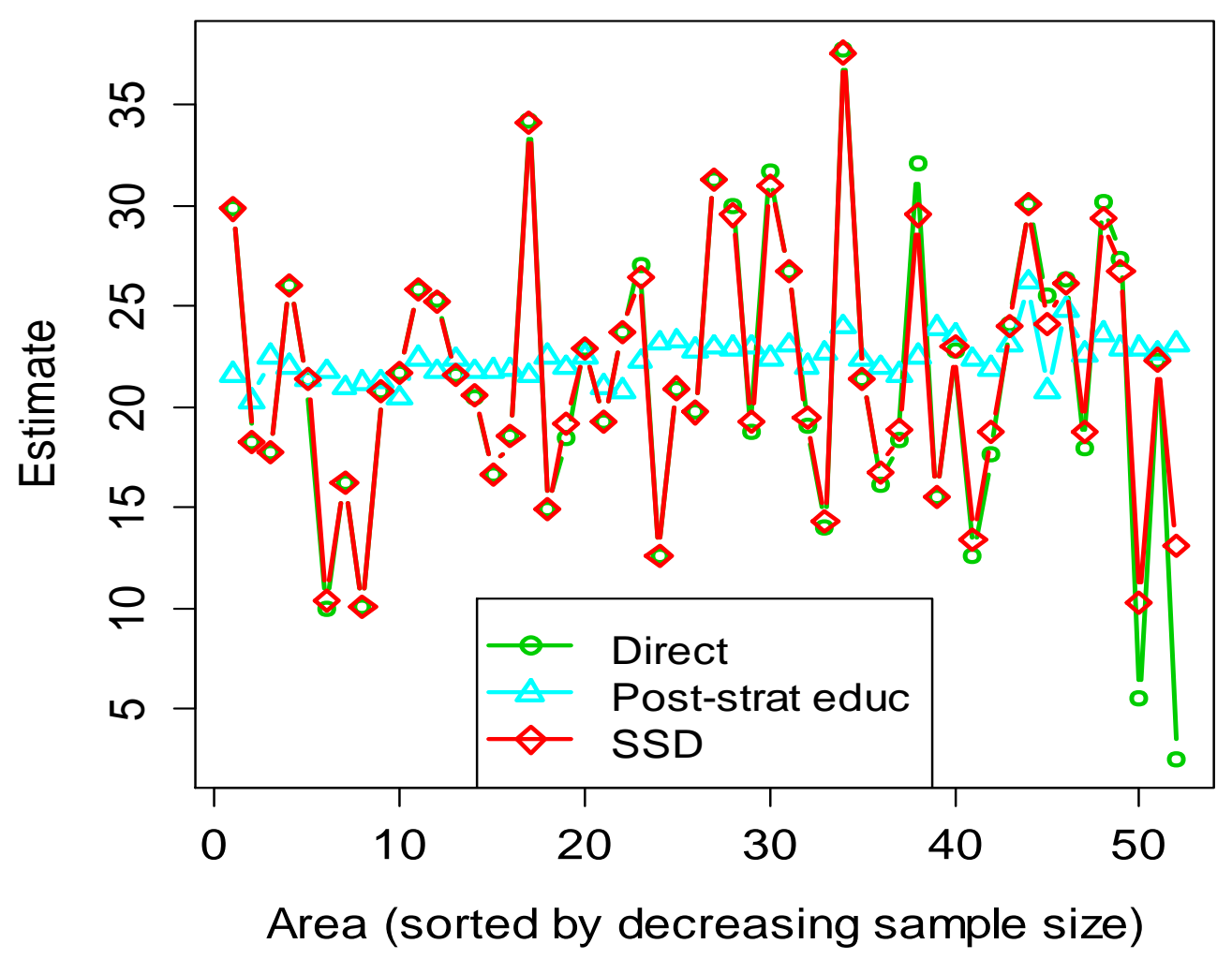

Figure 1: Sorted Combination of Direct (DIR), Post-stratified synthetic and Sample size dependent (SSD) according to each province

These estimates are plotted in the Figure for each province (area), with provinces sorted by decreasing sample size. This shows that direct estimates and SSD estimates are very similar. The estimated value of these two methods are fluctuately decreases as sample size decreases and they are slightly more unstable. It is noticeable that when sample size is large the SSD estimator treated as a direct estimator, but it is increases when sample size was small. However, the poststratified synthetic estimates appear to be too stable, giving practically the same values for all provinces. From the result it can be conclude that Direct estimator and SSD estimator have a similar impact on estimation procedure and Post-stratified Synthetic estimator is the best estimator than Direct and SSD estimator. 
Table 3: Descriptive statistics of Direct, Post-stratified synthetic and SSD estimate

\begin{tabular}{|c|c|c|c|}
\hline \multicolumn{4}{|c|}{ Descriptive statistics } \\
\hline Estimator & Direct & $\begin{array}{c}\text { Post-stratified } \\
\text { synthetic }\end{array}$ & SSD \\
\hline Minimum & 2.54 & 20.28 & 10.03 \\
\hline Maximum & 37.72 & 26.17 & 37.58 \\
\hline Mean & 21.3766 & 22.3575 & 21.6464 \\
\hline Standard Error (SE) & .98729 & .14871 & .86618 \\
\hline Standard deviation & 7.11945 & 1.07233 & 6.24609 \\
\hline
\end{tabular}

Above Table 3 depicted a descriptive comparison among the three estimator namely Direct estimator, Post-stratified Synthetic estimator and Sample Size Dependent (SSD) estimator. The Direct estimated values ranging from 2.54 to 37.72 , which is approximately similar to the SSD estimated value ranging from 10.03 to 37.03 . But in SSD the minimum estimated value (10.03) is greater than the Direct estimated value (2.54), in this sense SSD estimator have a great impact in small area estimation. The Post-stratified Synthetic estimates are ranging from 20.28 to 26.17. The mean value of these three estimators are approximately similar to each other.

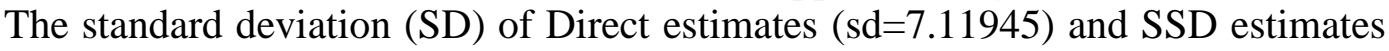
$(\mathrm{sd}=6.24609)$ are approximately similar, but they are greater than the standard deviation (SD) of Post-stratified Synthetic estimates ( $\mathrm{sd}=1.07233)$. Therefore, from Table 3, it can be conclude that among these three estimator Post-stratified Synthetic estimator is the best estimator than Direct and SSD estimator.

The estimation of nonlinear parameters of EB estimators based on BHF model provided by Battese et al. (1988). The values of the auxiliary variables in the model are needed for each out-of-sample unit. We use the sample data from all the provinces to fit the model and compute EB estimates and corresponding MSE estimates for all the provinces. For these selected provinces, the data set Xoutsamp contains the values for each out-of-sample individual of the considered auxiliary variables, which are the categories of education level and of labor status, defined exactly as in the data set incomedata. MSE estimates of the EB estimators under BHF model can be obtained using the parametric bootstrap method for finite populations introduced by González-Manteiga et al. (2008). Again, these data have been obtained by simulation.

To calculate EB estimates of the poverty incidences under BHF model for log (income + constant) for all provinces to fulfill the normality assumption. The list fit of the output gives information about the fitting process. The resulted linear mixed effects model fit by REAL method are depicted below, whether we find that all the auxiliary variables are significant (see Fixed effect table) and the correlation among the variables are independent (Fixed effects correlation table).

REML criterion at convergence: 18966.7 


\begin{tabular}{|l|c|c|}
\hline \multicolumn{3}{|c|}{ Random effects } \\
\hline Groups & Variance & Standard Dev. \\
\hline Dom (intercept) & 0.008904 & 0.09436 \\
\hline \multicolumn{1}{|c|}{ Residual } & 0.174676 & 0.41794 \\
\hline $\begin{array}{l}\text { Number of observation: } 17199 \\
\text { Number of domain : 52 }\end{array}$ \\
\hline
\end{tabular}

\begin{tabular}{|c|c|c|c|c|}
\hline \multicolumn{5}{|c|}{ Fixed effects } \\
\hline & Estimate & Std. Error & $\mathrm{t}$ value & $\mathrm{p}$-value \\
\hline Xs(Intercept & 9.505176 & 0.014385 & 660.8 & 0.00 \\
\hline Xseduc1 & -0.124043 & 0.007281 & -17.0 & 0.00 \\
\hline Xseduc3 & 0.291927 & 0.010366 & 28.2 & 0.00 \\
\hline Xslabor1 & 0.145985 & 0.006916 & 21.1 & 0.00 \\
\hline Xslabor2 & -0.081624 & 0.017083 & -4.8 & 0.00 \\
\hline
\end{tabular}

\begin{tabular}{|l|c|c|c|c|}
\hline \multicolumn{5}{|c|}{ Correlation of fixed effects } \\
\hline & Xs(In) & Xsedc1 & Xsedc3 & Xslabor1 \\
\hline Xseduc1 & -0.212 & & & \\
\hline Xseduc1 & -0.070 & 0.206 & & \\
\hline Xslabor1 & -0.199 & 0.128 & -0.228 & \\
\hline Xslabor2 & -0.079 & 0.039 & -0.039 & 0.168 \\
\hline
\end{tabular}

Checking model assumptions is crucial since the optimality properties of the EB estimates depend on the extent to which those assumptions are true. We draw the usual residual plots to detect departures from BHF model for the transformed income. An index plot of residuals and a histogram are given below: 


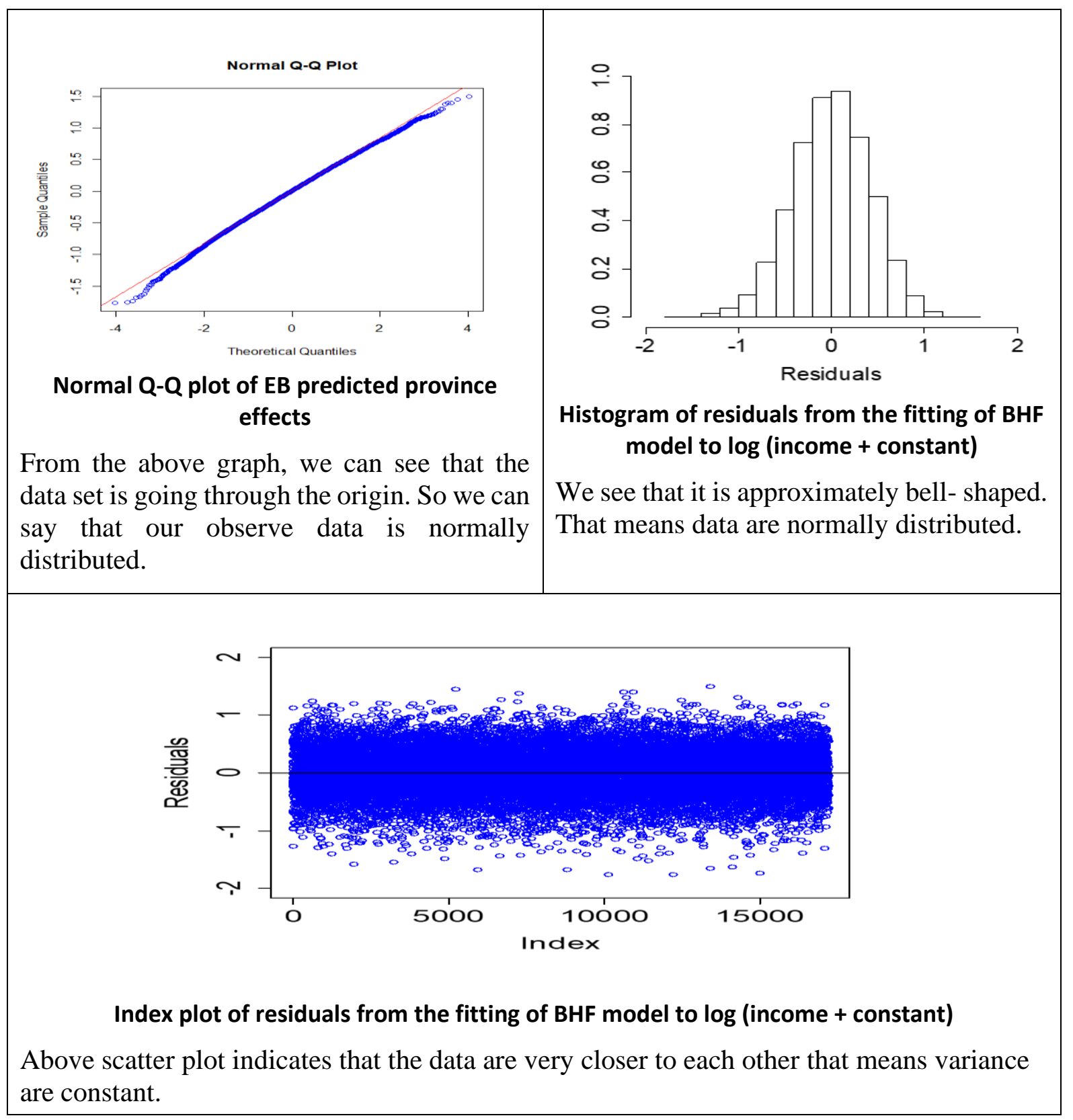

Figure 2: Checking model assumption 
Table 4: Comparison of Direct and Empirical Bayes (EB) estimates and their respective coefficient of variation

\begin{tabular}{|c|c|c|c|c|c|c|}
\hline $\begin{array}{l}\text { Province } \\
\text { Index }\end{array}$ & $\begin{array}{l}\text { Province } \\
\text { Name }\end{array}$ & $\begin{array}{c}\text { Sample } \\
\text { Size }\end{array}$ & Direct & CV. DIR & EB & CV. EB \\
\hline 1 & Alava & 96 & 0.255037 & 19.00367 & 0.335 & 7.226619 \\
\hline 2 & Albacete & 173 & 0.140592 & 21.6384 & 0.159711 & 12.93839 \\
\hline 3 & Alicante & 539 & 0.207851 & 10.48198 & 0.210909 & 5.402373 \\
\hline 4 & Almeria & 198 & 0.26764 & 15.28299 & 0.24101 & 7.834946 \\
\hline 5 & Avila* & 58 & 0.055122 & 46.35946 & 0.123793 & 24.96203 \\
\hline 6 & Badajoz & 494 & 0.215539 & 10.93958 & 0.201012 & 5.96569 \\
\hline 7 & Baleares & 634 & 0.099998 & 15.36549 & 0.11511 & 8.837025 \\
\hline 8 & Barcelona & 1420 & 0.298125 & 5.428952 & 0.289218 & 2.307215 \\
\hline 9 & Burgos & 168 & 0.214132 & 20.89156 & 0.188929 & 8.973926 \\
\hline 10 & Caceres & 282 & 0.270313 & 11.56369 & 0.331773 & 4.358747 \\
\hline 11 & Cadiz & 398 & 0.148874 & 14.7039 & 0.135251 & 8.6262 \\
\hline 12 & Castellon & 118 & 0.175982 & 20.37073 & 0.255 & 8.938094 \\
\hline 13 & CiudadReal & 250 & 0.209215 & 15.67395 & 0.20264 & 8.696991 \\
\hline 14 & Cordoba & 224 & 0.299757 & 13.12423 & 0.311161 & 5.926287 \\
\hline 15 & CorunaLa & 495 & 0.253475 & 9.73552 & 0.268444 & 3.99551 \\
\hline 16 & Cuenca & 92 & 0.263341 & 22.45527 & 0.247717 & 9.927219 \\
\hline 17 & Gerona & 142 & 0.183374 & 20.23291 & 0.222254 & 8.419048 \\
\hline 18 & Granada & 208 & 0.317273 & 12.74599 & 0.335529 & 5.548515 \\
\hline 19 & Guadalajara & 89 & 0.179082 & 23.64297 & 0.254157 & 9.667373 \\
\hline 20 & Guipuzcoa & 285 & 0.236905 & 13.48546 & 0.245158 & 6.101039 \\
\hline 21 & Huelva & 122 & 0.125834 & 25.45047 & 0.139508 & 16.48341 \\
\hline 22 & Huesca & 115 & 0.241076 & 20.14448 & 0.278 & 8.05499 \\
\hline 23 & Jaen & 232 & 0.312942 & 13.17392 & 0.284138 & 5.585637 \\
\hline 24 & Leon & 218 & 0.188016 & 15.97012 & 0.249541 & 6.945103 \\
\hline 25 & Lerida & 130 & 0.155596 & 24.88785 & 0.168231 & 12.96007 \\
\hline 26 & RiojaLa & 510 & 0.258118 & 9.527405 & 0.265843 & 3.809543 \\
\hline 27 & Lugo & 173 & 0.377187 & 15.10213 & 0.341387 & 5.242763 \\
\hline 28 & Madrid & 944 & 0.182182 & 8.996593 & 0.191716 & 3.688254 \\
\hline 29 & Malaga & 379 & 0.229185 & 11.93636 & 0.244908 & 5.155946 \\
\hline 30 & Murcia & 885 & 0.177032 & 9.31421 & 0.186565 & 4.323254 \\
\hline 31 & Navarra & 564 & 0.161908 & 11.37696 & 0.174574 & 5.835955 \\
\hline 32 & Orense & 129 & 0.227996 & 18.41902 & 0.306667 & 7.349597 \\
\hline 33 & Oviedo & 803 & 0.26064 & 8.03322 & 0.268319 & 3.274346 \\
\hline 34 & Palencia* & 72 & 0.301661 & 23.80085 & 0.274306 & 11.25716 \\
\hline 35 & PalmasLas & 472 & 0.166518 & 13.85587 & 0.150212 & 7.020753 \\
\hline 36 & Pontevedra & 448 & 0.185491 & 13.04047 & 0.161652 & 7.475342 \\
\hline 37 & Salamanca & 164 & 0.161045 & 18.61741 & 0.199634 & 10.25905 \\
\hline 38 & Tenerife & 381 & 0.184296 & 11.14808 & 0.249711 & 4.648279 \\
\hline
\end{tabular}




\begin{tabular}{|c|c|c|c|c|c|c|}
\hline 39 & Santander & 434 & 0.342444 & 9.487491 & 0.351866 & 3.26399 \\
\hline 40 & Segovia* $^{*}$ & 58 & 0.22262 & 25.33449 & 0.283448 & 10.66742 \\
\hline 41 & Sevilla & 482 & 0.20503 & 10.35226 & 0.231784 & 4.650448 \\
\hline 42 & Soria* $^{*}$ & 20 & 0.025412 & 99.97815 & 0.1175 & 42.7319 \\
\hline 43 & Tarragona & 134 & 0.320354 & 15.40193 & 0.414328 & 5.333037 \\
\hline 44 & Teruel* & 72 & 0.273642 & 24.57017 & 0.315556 & 9.257185 \\
\hline 45 & Toledo & 275 & 0.125534 & 16.98341 & 0.149673 & 9.837353 \\
\hline 46 & Valencia & 714 & 0.213607 & 9.693081 & 0.228852 & 3.890675 \\
\hline 47 & Valladolid & 299 & 0.192923 & 16.64643 & 0.157492 & 9.689178 \\
\hline 48 & Vizcaya & 524 & 0.216945 & 10.21295 & 0.22063 & 5.150919 \\
\hline 49 & Zamora & 104 & 0.300274 & 20.06599 & 0.274712 & 8.936914 \\
\hline 50 & Zaragoza & 564 & 0.100346 & 15.63731 & 0.112996 & 8.187835 \\
\hline 51 & Ceuta & 235 & 0.197248 & 16.93905 & 0.189915 & 8.724863 \\
\hline 52 & Melilla & 180 & 0.191091 & 18.00719 & 0.216833 & 7.904507 \\
\hline
\end{tabular}

Above table shows that most of the estimated value of Direct estimation for each province less than the estimated value obtain by EB estimation. Coefficient of variation in each province for direct estimate is greater than the coefficient of variation $(\mathrm{CV})$ of EB estimate. It can be visually shown in Figure 3 and Figure 4.

*indicate 5 selected province with small sample size. The table shows that the estimated value of Direct (DIR) estimators for four provinces with small sample size namely Avila, Segovia, Soria, Teruel poverty incidence lie under EB estimates. Additionally that the differences are large in three provinces (Avila, Segovia, Soria), and are small in one of them (palencia). The above table also shows that the estimated C.Vs of Direct (DIR) for poverty incidence estimators are noticeably larger than those of Empirical Bayes (EB) estimators in all provinces. Such that we can say that EB estimate is better than direct estimation. 


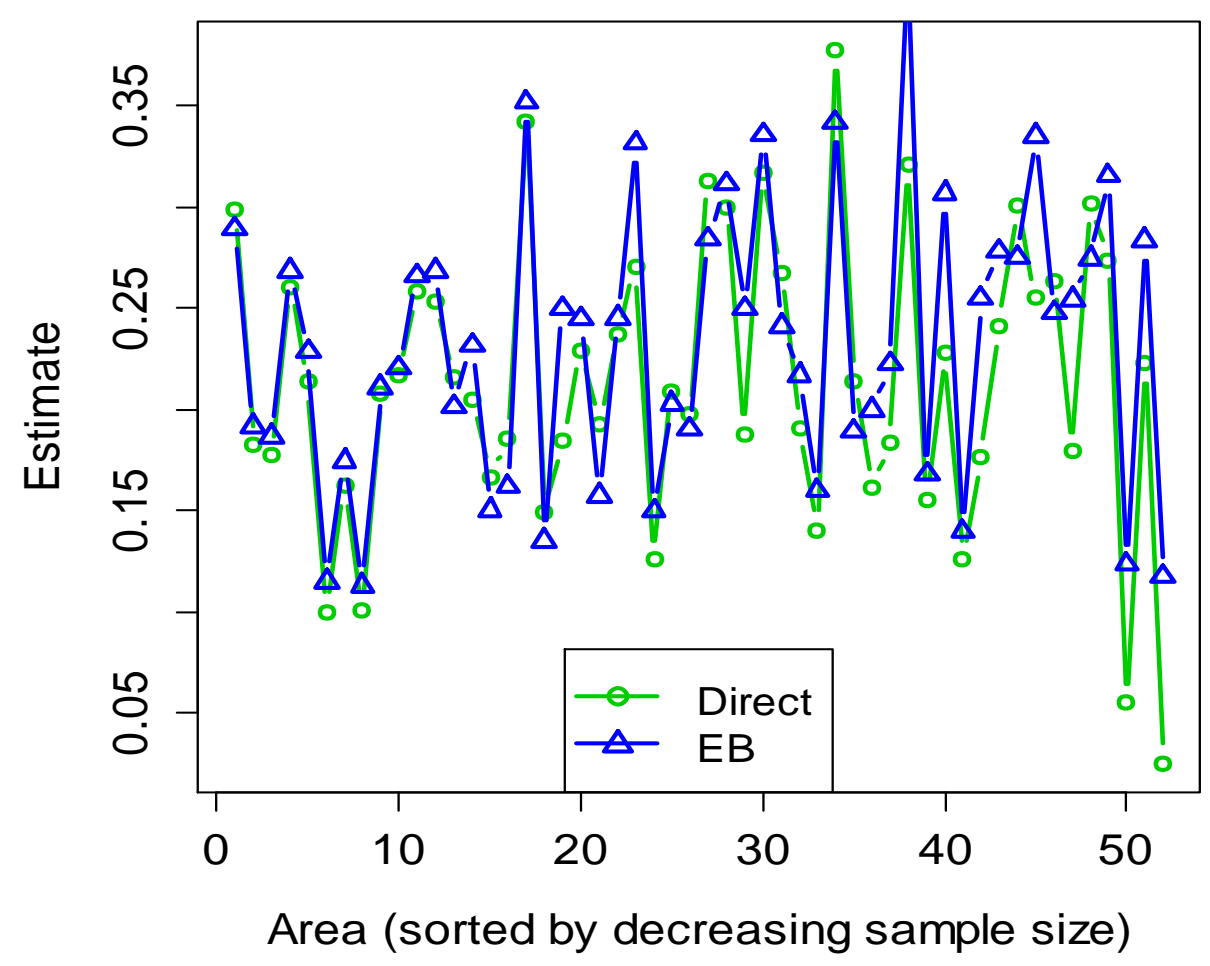

Figure 3: The estimated poverty incidence for Direct (DIR) and EB estimates

The figures above showed that most of the direct estimates for poverty incidence lie under Empirical Bayes (EB) estimator for all selected provinces. 


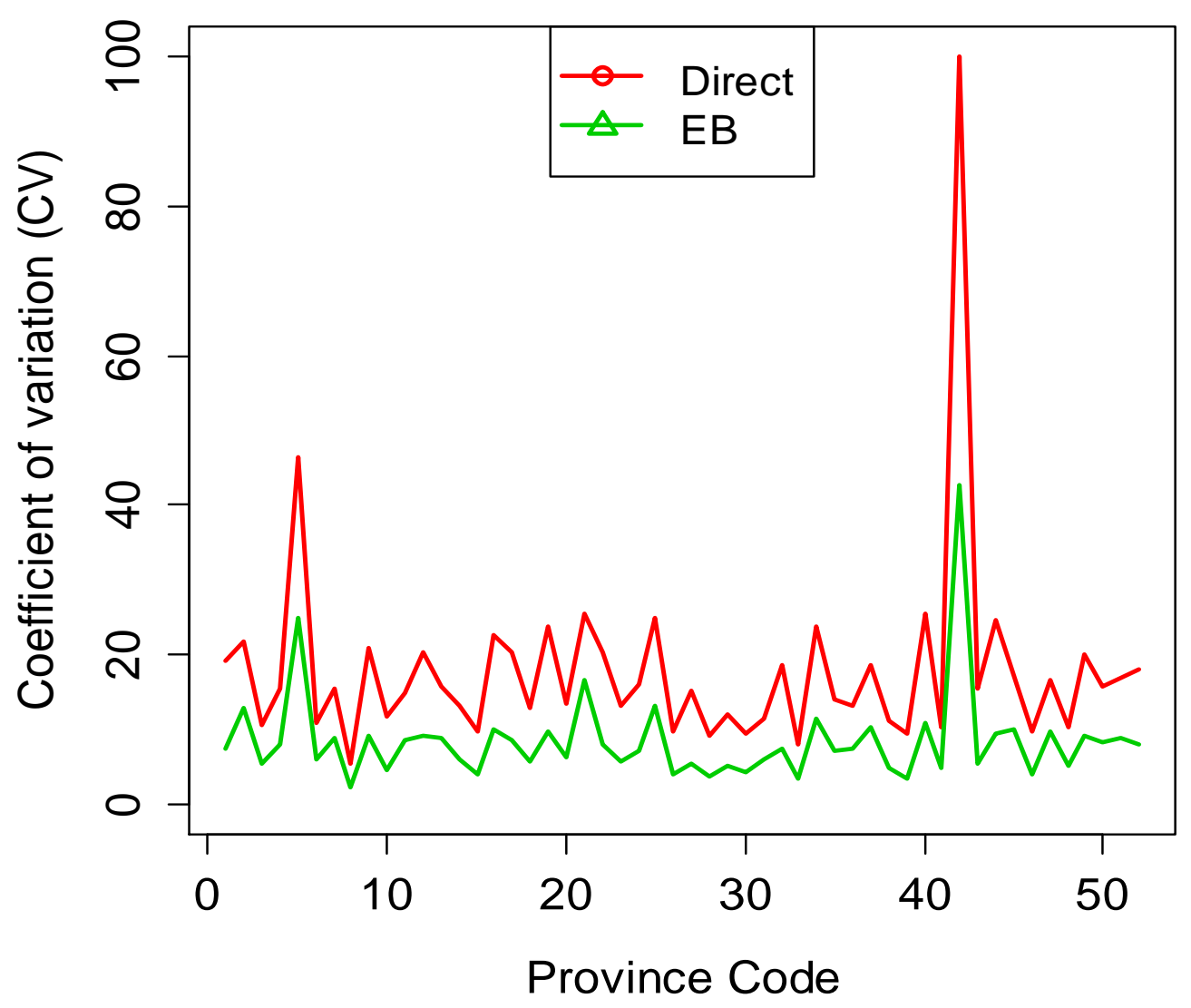

Figure 4: Estimated C.Vs of Direct (DIR) and EB for each area

The above graph shows that the estimated C.Vs of Direct (DIR) estimators are noticeably larger than those of Empirical Bayes (EB) estimators in all provinces. From Figure 3 and Figure 4, it is clear that model based estimator such as Empirical Bayes (EB) estimator is one of the most efficient estimator than direct estimator in the case of small area estimation. 


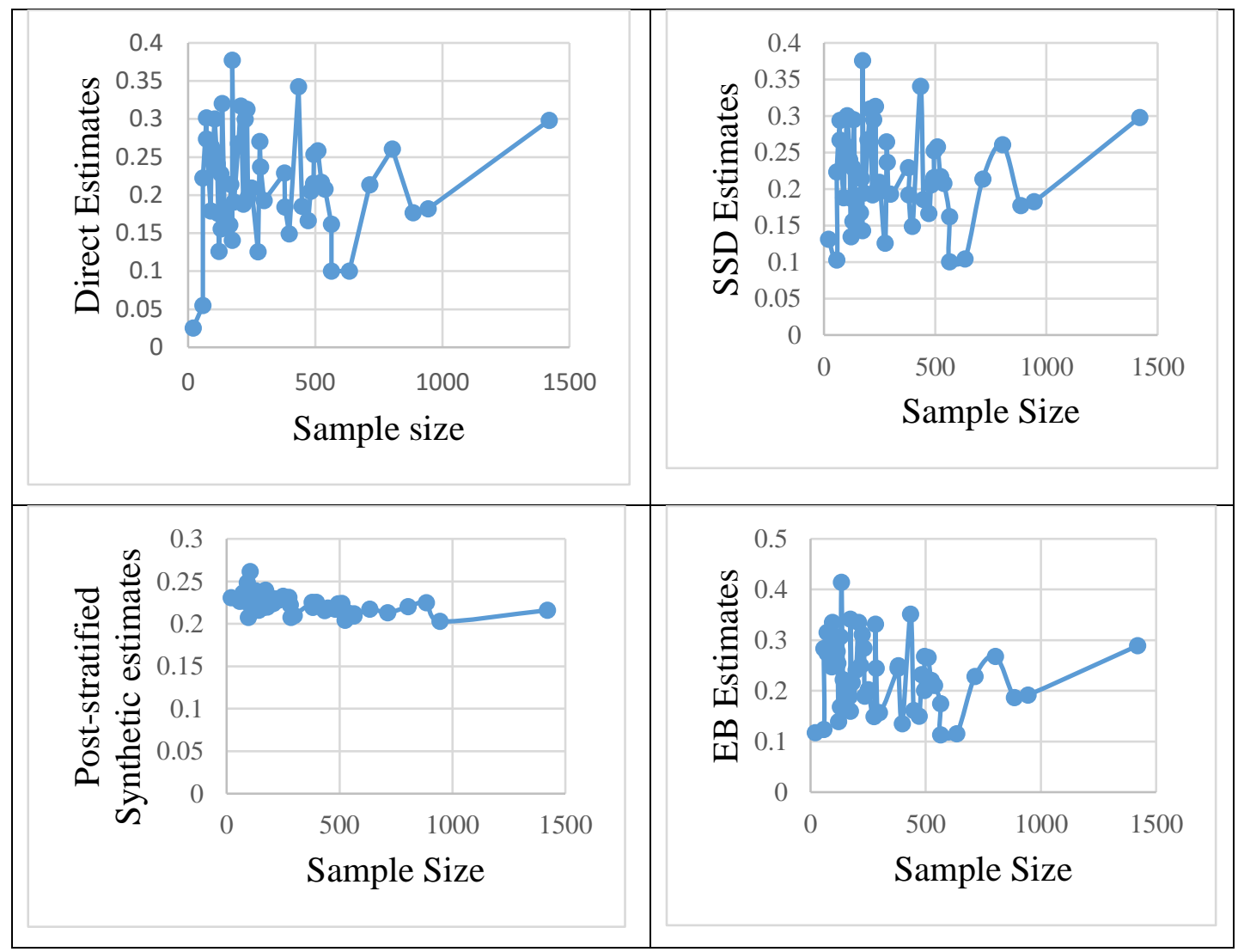

Figure 5: Scatter plot of Direct, SSD, Post-stratified synthetic and EB estimates according to sample size

From the Figure 5 it can be seen that scatter plot of direct estimates (Range: 0.025412-0.377187), SSD estimates (Range: .100346-.375823) and EB estimates (Range: 0.11299-0.41432) according to the sample size estimated value are fluctuately increasing as sample size increases, they are unstable (Range: 0.025412$0.377187)$ and they are approximately same. But in the case of post-stratified synthetic estimated values are stable (Range: 0.202825-0.261705). Comparing to the direct estimates with indirect estimates (post-stratified synthetic, SSD, EB) it can be shown that the estimated value of indirect estimates are greater than direct estimated values as if there is small sample size. 


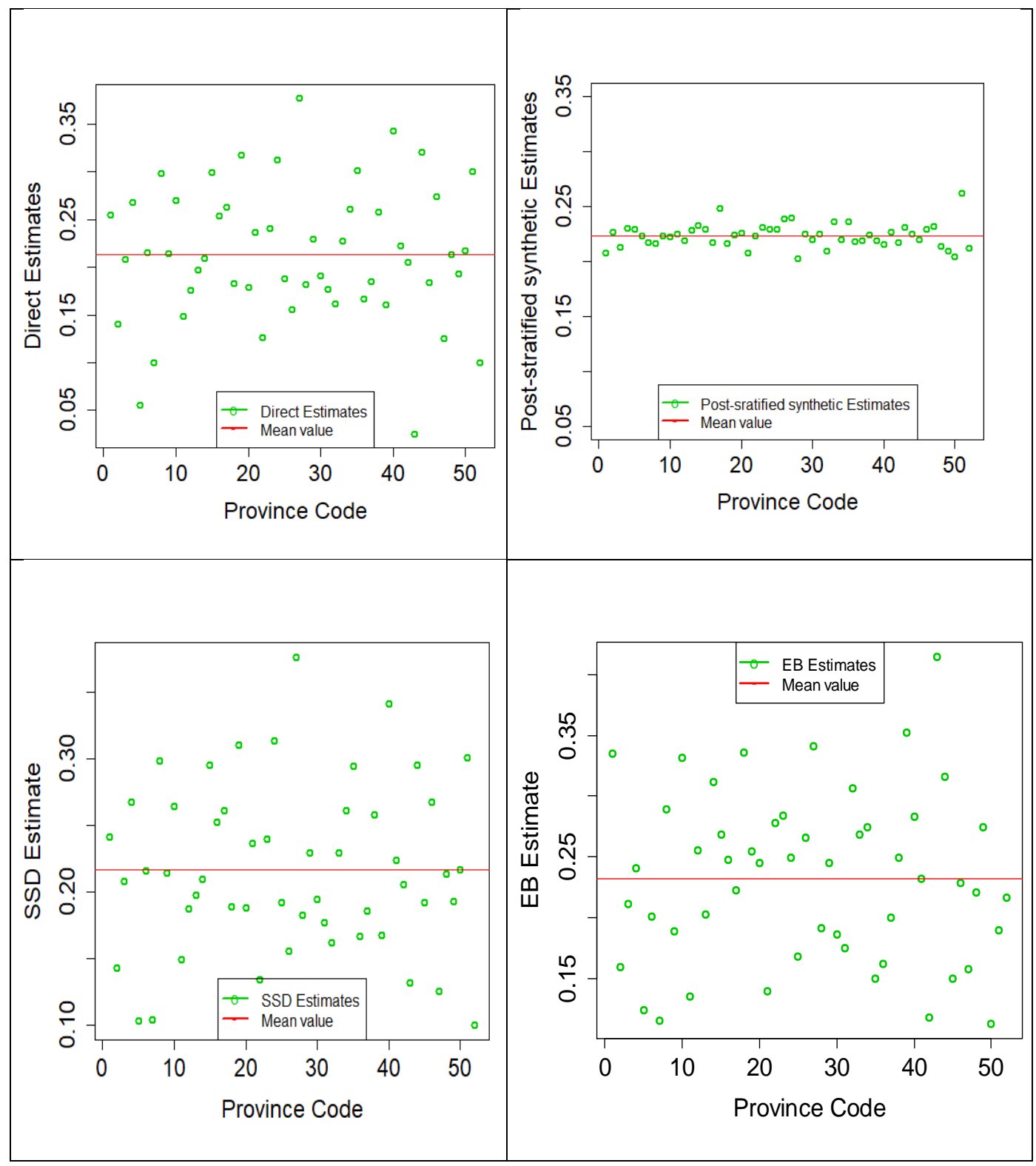

Figure 6: Scatter plot of Direct, post-stratified synthetic, SSD and EB estimates according to province code

Figure 6 indicates that the estimated value of Direct, SSD and EB estimator have a great distance from their mean value and the estimated value of Post-stratified Synthetic estimator are closer to their mean value and they are approximately stable. 


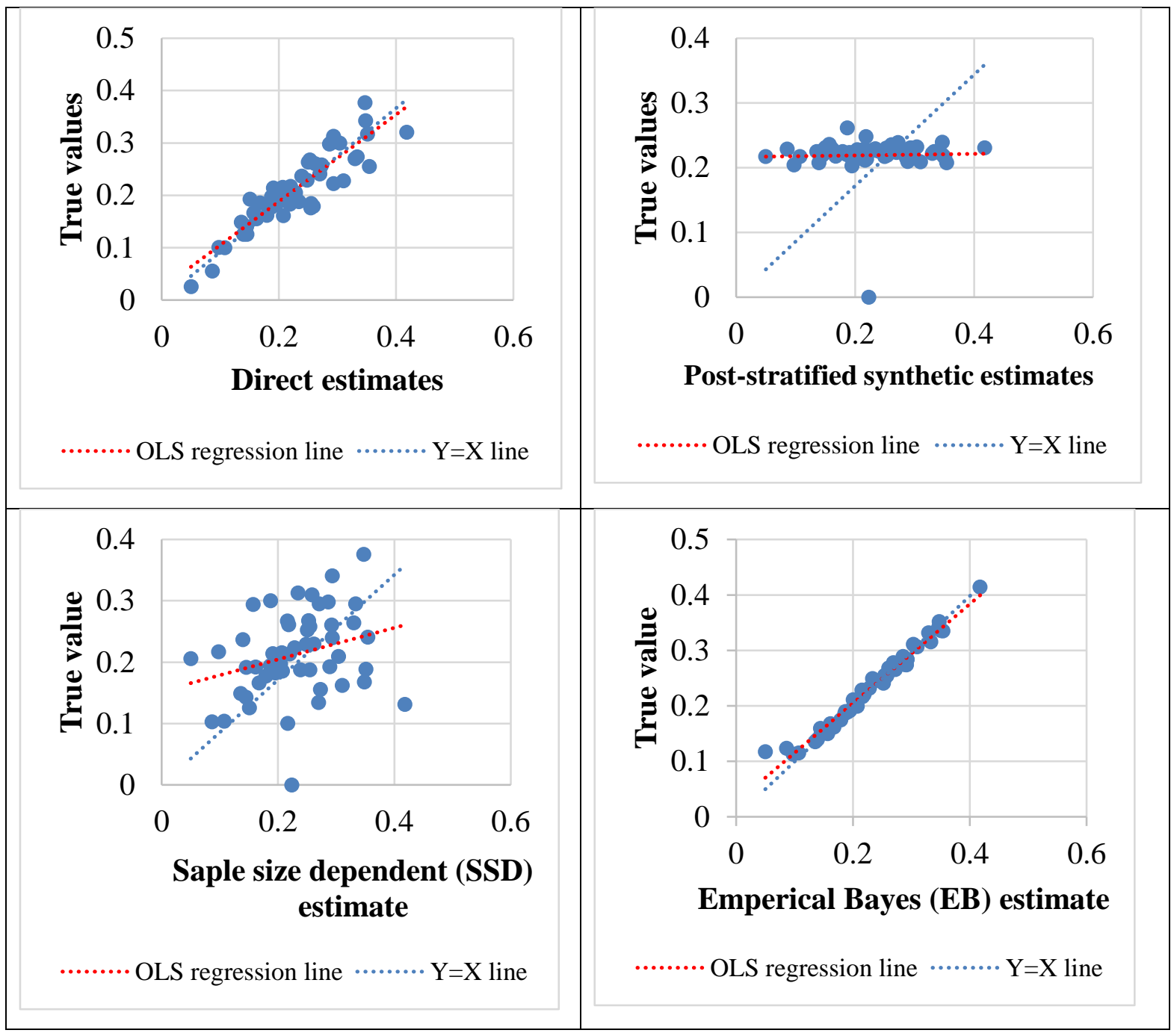

Figure 7: Bias scatter plot between True values and Direct, post-stratified synthetic and EB estimates

The scatter plot of true value (on the Y-axis) against direct estimates and EB estimates (on the $\mathrm{X}$-axis) displayed a regression line close to the $\mathrm{Y}=\mathrm{X}$ line. Here the slope coefficient estimate was near to 1 and intercept was not significantly differ from zero (0), indicating that there is no evidence to reject the hypothesis of lack bias for the direct estimate. That means, it can be conclude that Direct estimates and EB estimates are approximately unbiased. The main difference is that the value points in EB estimator are situated exactly in a straight line but the points of direct estimator have a significance distance from the straight line.

The scatter plot of true value (on the Y-axis) against post-stratified synthetic estimates and SSD estimates (on the $\mathrm{X}$-axis) displayed a regression line that are not close to the $\mathrm{Y}=\mathrm{X}$ line. Here the slope coefficient estimate was not near to 1 and intercept was significantly differ from zero (0), indicating that there is an evidence 
to reject the hypothesis of lack bias for the post-stratified synthetic estimate. That means, it can be conclude that post-stratified synthetic estimates and SSD estimates are extreme biased.

\section{Conclusion}

Our estimated results shown that direct estimates and sample size dependent (SSD) estimates are very similar. The estimated value of these two methods are fluctuately decreases as sample size decreases and they are slightly more unstable. It is noticeable that when sample size is large the SSD estimator treated as a direct estimator, but it is increases when sample size was small. However, the poststratified synthetic estimates appear to be too stable, giving practically the same values for all provinces. But direct estimator is approximately unbiased but SSD and Post-stratified synthetic estimator is extreme biased. EB estimator depicted that most of the estimated value of Direct estimation for each province less than the estimated value obtain by EB estimation. Coefficient of variation in each province for direct estimate is greater than the coefficient of variation (CV) of EB estimate and it can also be shown that EB estimator is approximately unbiased. Such that we can say that EB (model based) estimate is better estimation method in the case of small area estimation. That's why, it is impossible to think any research work without knowing the SAE technique in the present world. 


\section{References}

[1] Arora, V. and Lahiri, P. (1997). On the superiority of the Bayesian method over the BLUP in small area estimation problems. Statistica Sinica, 7, 10531063.

[2] Rahmn, A. (2008). A Review of Small Area Estimation Problems and Methodological Developments. Australia. NATSEM, University of Canberra.

[3] Chandra, H., Aditya, K. \& Sud, U. C. (2018). Localised estimates and spatial mapping of poverty incidence in the state of Bihar in India - An application of small area estimation techniques. PLoS ONE, 13, 6, e0198502.

[4] Datta, G. S., Rao, J. N. K. and Smith, D. D. (2005). On measuring the variability of small area estimators under a basic area level model. Biometrika, 92,1, 183-196.

[5] Drew, D., Singh, M.P. \& Choudhry, G.H. (1982). Evaluation of small area estimation techniques for the Canadian Labour Force Survey. Survey Methodology, 8, 17-47.

[6] Drew, D., Singh, M.P. and Choudhry, G.H. (1982). Evaluation of small area estimation techniques for the Canadian Labour Force Survey. Survey Methodology, 8, 17-47.

[7] El-Yasha, S. R., Rizky, M., Wibowo, T. W. \& Sudaryatno, (2019). Spatial Analysis of Poverty and Happiness Profiles in Special Region of Yogyakarta Using Small Area Estimation Method. The International Archives of the Photogrammetry, Remote Sensing and Spatial Information Sciences, Volume XLII-4/W16.

[8] European Commission. "Small Area Methods for Poverty and Living Condition Estimates Project", http://www.sample-project.eu/.

[9] Ghosh, M. \& Rao, J.N.K. (1994). Small area estimation: an appraisal. Statistical Science, 9, 55-93.

[10] Kamal El Saied, M. M., Talat, A. A. and El Gohary, M. M. (2019). Small Area Procedures for Estimating Income and Poverty in Egypt. Asian Journal of Probability and Statistics, 4, 1, 1-17.

[11] Marchetti, S., Giusti, C., Pratesi1, M. Salvati, N., Giannotti, F., Pedreschi, D., Rinzivillo, S., Pappalardo, L., and Gabrielli, L. (2015). Small Area ModelBased Estimators Using Big Data Sources. Journal of Official Statistics, 31, 2, 263-281. http://dx.doi.org/10.1515/JOS-2015-0017

[12] Molina, I. \& Marhuenda, Y. (1982). sae: An R package for Small Area Estimation. R Journal, Under revision.

[13] Molina, I. and Marhuenda, Y. (2015). sae: An R Package for Small Area Estimation. The R Journal Vol. 7/1.

[14] Nájera Catalán, H.E., Fifita, V.K. and Faingaanuku, W. (2020). Small-Area Multidimensional Poverty Estimates for Tonga 2016. Drawn from a Hierarchical Bayesian Estimator. Appl. Spatial Analysis 13, 305-328. https://doi.org/10.1007/s12061-019-09304-8 
[15] Pusponegoro, N. H. and Rachmawati, R. N. (2018). Spatial Empirical Best Linear Unbiased Prediction in Small Area Estimation of Poverty. Science Direct, 135, 712-718.

[16] Pusponegoro, N. H., Djuraidah, A., Fitrianto A. and Sumertajaya, I. M. (2019). Geo-additive Models in Small Area Estimation of Poverty. Journal of Data Science and Its Applications, 2, 1, 11-18.

[17] Rao, J. N. K. and Molina, I. (2015). Small area estimation 2nd edition. New Jersey, John Wiley \& Sons.

[18] Rao, J.N.K. (2003). Small Area Estimation. Wiley, London.

[19] Suhartini T., Sadik, K. and Indahwati, (2016). Small area estimation (SAE) model: Case study of poverty in West Java Province. AIP Conference Proceedings 1707, 080016. https://doi.org/10.1063/1.4940873

[20] Sundara, V. Y., Kurnia, A. and Sadik, K. (2017). Clustering Information of Non-Sampled Area in Small Area Estimation of Poverty Indicators. IOP Conference Series: Earth and Environmental Science, 58, 012020. https://doi.org/10.1088/1755-1315/58/1/012020

[21] Szymkowiak, M., Młodak, A. and Wawrowski, L. (2017). Mapping Poverty at the Level of Subregions in Poland Using Indirect Estimation. STATISTICS IN TRANSITION new series, 18, 4, 609-635.

[22] You, Y. and Chapman, B. (2006). Small area estimation using area level models and estimated sampling variances. Survey Methodology, 32, 97-103. 